\title{
DESIGN AND IMPLEMENTATION OF DIGITAL FILTER BANK TO REDUCE NOISE AND RECONSTRUCT THE INPUT SIGNALS
}

\author{
Kawser Ahammed, Md. Ershadullah, Md. Rakebul Islam Heru and Saiful Islam \\ Department of Electrical \& Electronic Engineering \\ (Former Applied Physics, Electronics \& Communication Engineering), \\ University of Dhaka, Dhaka, Bangladesh
}

\begin{abstract}
The main theme of this paper is to reduce noise from the noisy composite signal and reconstruct the input signals from the composite signal by designing FIR digital filter bank. In this work, three sinusoidal signals of different frequencies and amplitudes are combined to get composite signal and a low frequency noise signal is added with the composite signal to get noisy composite signal. Finally noisy composite signal is filtered by using FIR digital filter bank to reduce noise and reconstruct the input signals.
\end{abstract}

\section{KEYWORDS}

Digital Filter Bank, Noise, LMS Filter, LMS Algorithm, Composite Signal

\section{INTRODUCTION}

Over the past two decades, the research on efficient design of filter banks has received considerable attention in numerous fields such as speech coding, scrambling, image processing etc. [1-2]. At the same time design of filter banks has received attention on noise reduction. So noises in any digital signals significantly hamper the actual performance of signals at the desired output [3-4]. So filter banks are required to reduce these noises. A filter bank is nothing but a group of parallel low pass, band pass and high pass filters [5] that separate the input signal into multiple components, each one carrying a single frequency subband of the original signal [6]. The process of decomposition performed by the filter bank is called analysis. The reconstruction process is called synthesis, meaning reconstitution of a complete signal resulting from the filtering process [7]. Filter banks are generally classified as two types, analysis filter banks and synthesis filter banks [8]. An analysis filter bank comprises of filters, with system transfer functions $\left\{\mathrm{H}_{\mathrm{k}}(\mathrm{z})\right\}$, where $\mathrm{k}=0,1, \ldots, \mathrm{M}-1$; arranged in a parallel bank as shown in Figure 1. On the contrary, a synthesis filter bank comprises of a set of filters with system transfer functions $\left\{\mathrm{G}_{\mathrm{k}}(\mathrm{z})\right\}$, where $\mathrm{k}=0,1, \ldots, \mathrm{M}-1$; arranged as shown in Figure 2, with corresponding inputs $\left\{\mathrm{y}_{\mathrm{k}}(\mathrm{m})\right\}$.The outputs of the filters are summed to form the synthesized signal $\tilde{x}(n)$. The blocks with arrows pointing downwards in Figure1 indicate down sampling by factor $\mathrm{N}$, and the blocks with arrows pointing upwards in Figure 2 indicate up sampling by $\mathrm{N}$. 


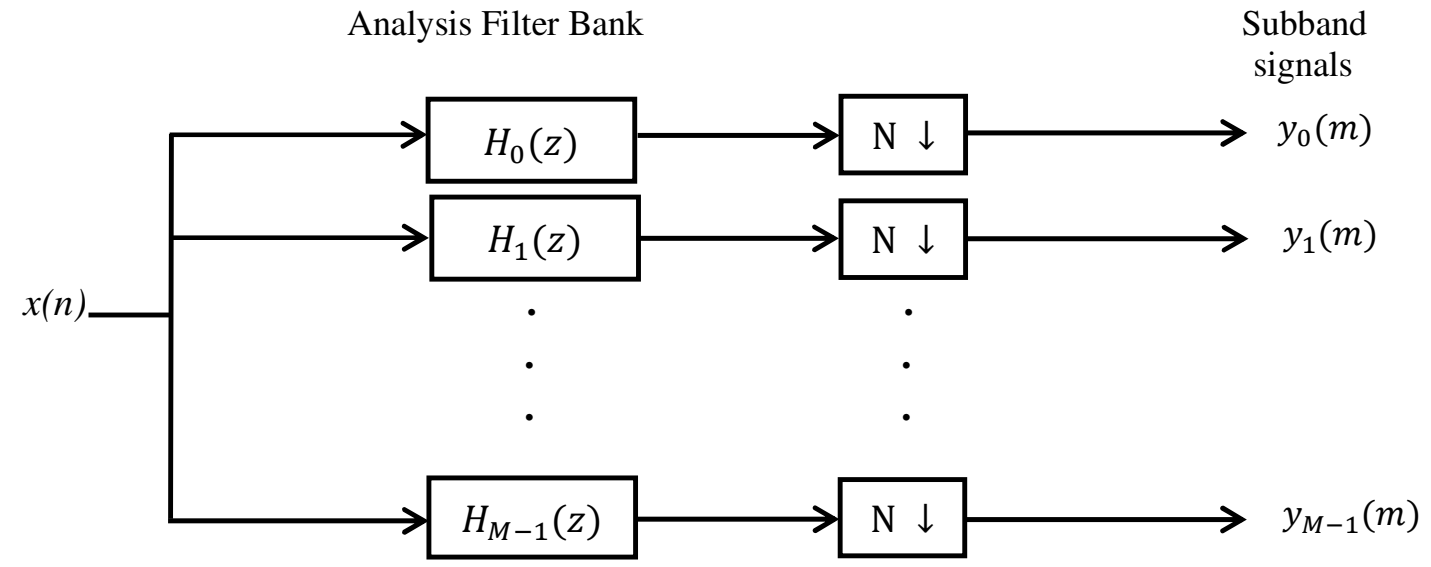

Figure 1. Analysis filter bank, adapted from Ref. [1]

Sub sampling by $\mathrm{N}$ means that only every $\mathrm{N}$-th sample is taken. This operation serves to reduce or eliminate redundancies in the $\mathrm{M}$ subband signals. Up sampling by $\mathrm{N}$ means the insertion of $\mathrm{N}$ 1 consecutive zeros between the samples. This allows us to recover the original sampling rate. There are many applications of filter banks such as graphic equalizer, signal compression, bank of receiver, noise reduce etc.

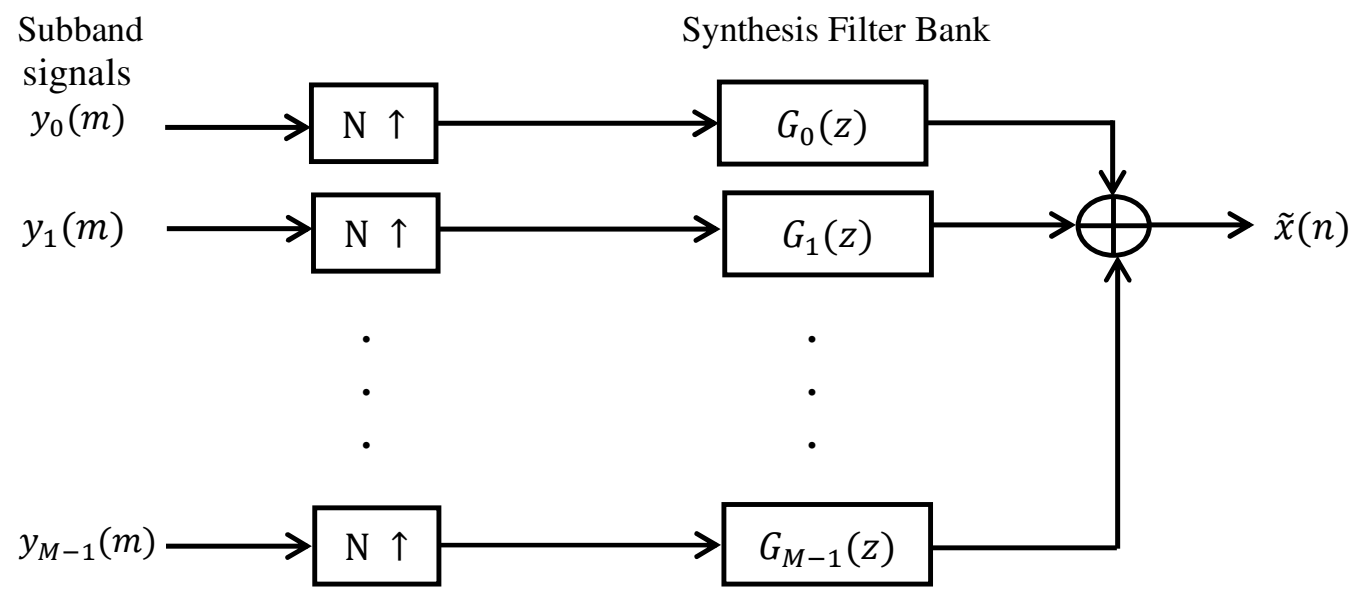

Figure 2. Synthesis filter bank, adapted from Ref. [1]

\section{EXAMPLE OF A FILTER BANK}

In this section, a two channel filter bank adapted from ref. [9] has been described. Figure 3 shows such kind of two channel filter bank. It is convenient to analyse the filter bank in the Z-domain. Therefore, by using Z-transform the expressions for the various intermediate signals in figure 3 are given by

$$
\begin{aligned}
& R_{l}(z)=H_{l}(z) U(z) \\
& S_{l}(z)=\frac{1}{2}\left\{R_{l}\left(z^{\frac{1}{2}}\right)+R_{l}\left(-z^{\frac{1}{2}}\right)\right\}
\end{aligned}
$$




$$
W_{l}(z)=S_{l}\left(z^{2}\right)
$$

for $l=0,1$. After performing some algebra, the following equation is obtained

$$
W_{l}(z)=\frac{1}{2}\left\{R_{l}(z)+R_{l}(-z)\right\}=\frac{1}{2}\left\{H_{l}(z) U(z)+H_{l}(-z) U(-Z)\right\}
$$

Now the reconstructed output of the filter bank is attained by

$$
V(z)=G_{0}(z) W_{0}(z)+G_{1}(z) W_{1}(z)
$$

Substituting equation (4) in equation (5), the output of the filter bank is obtained by

$$
\begin{gathered}
V(z)=\frac{1}{2}\left\{H_{0}(z) G_{0}(z)+H_{1}(z) G_{1}(z)\right\} U(z)+\frac{1}{2}\left\{H_{0}(-z) G_{0}(z)\right. \\
\left.+H_{1}(-z) G_{1}(z)\right\} U(-z)
\end{gathered}
$$

The first term in the above equation describes the transmission of the signal $U(\mathrm{z})$ through the system, while the second term describes the aliasing component at the output. The above equation can be compactly represented as

$$
V(z)=D(z) U(z)+B(z) U(-z)
$$

Where $D(z)=\frac{1}{2}\left\{H_{0}(z) G_{0}(z)+H_{1}(z) G_{1}(z)\right\} U(z)$

is called the distortion transfer function, and

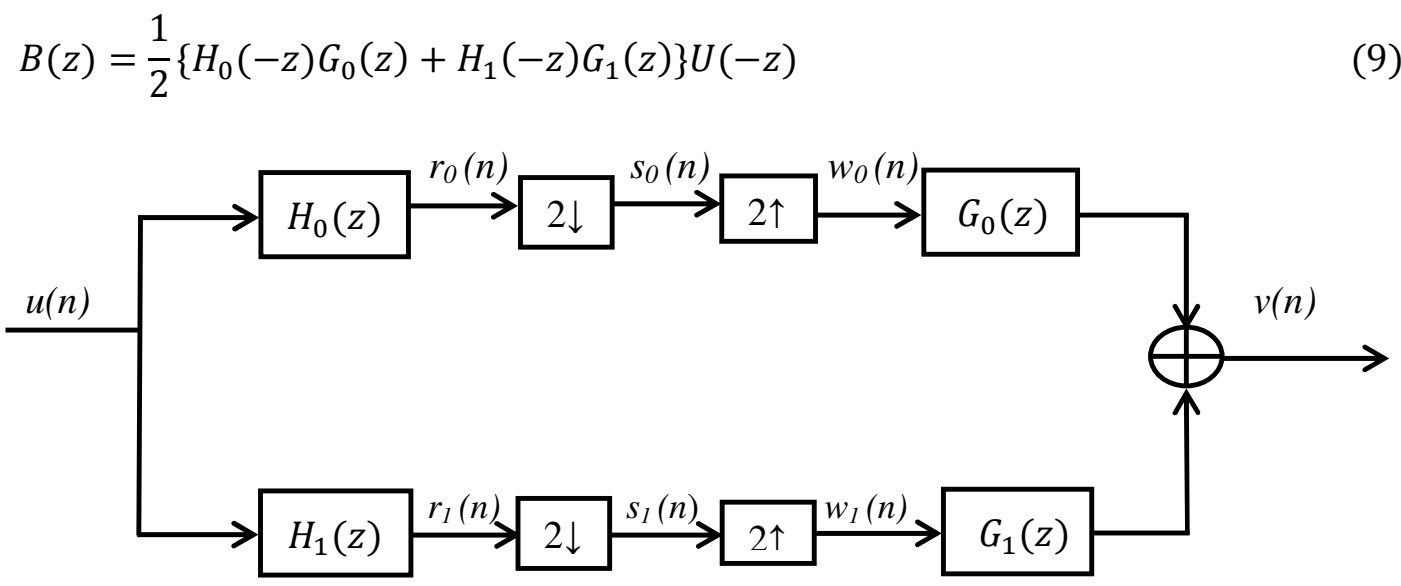

Figure 3. Two channel filter bank, adapted from Ref. [1]

\section{METHODOLOGY}

At the first step, amplitude, frequency and sample time of three sinusoidal signals, denoted by A1, $\mathrm{B} 1$ and $\mathrm{C} 1$, are fixed to form composite signal and a random source is considered to get random signal. The random source acts as a noise source. The block parameters of three sinusoidal signals are shown in Figure 4. 
Signal \& Image Processing : An International Journal (SIPIJ) Vol.6, No.2, April 2015

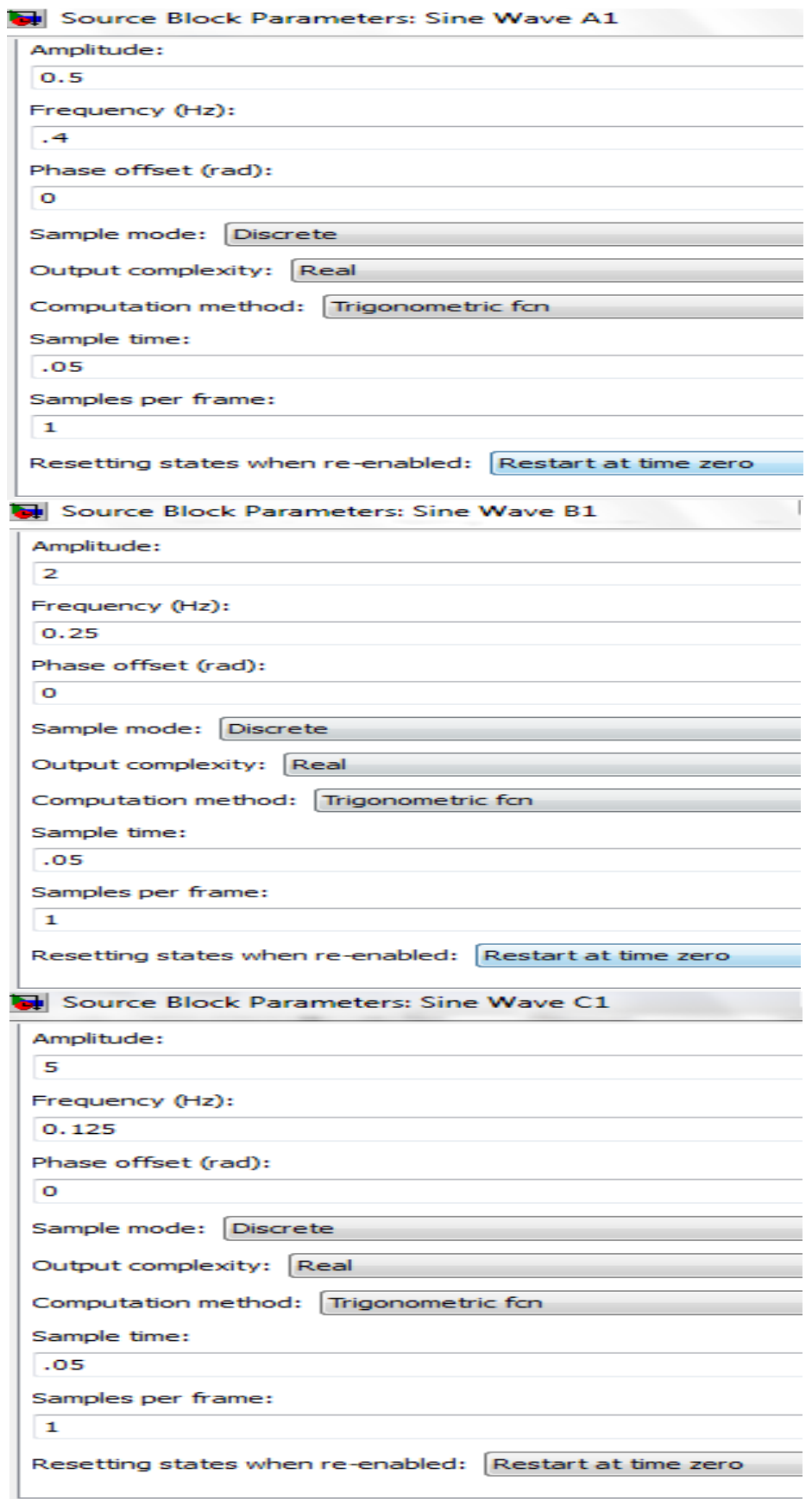

Figure 4. Block parameters of three sinusoidal signals A1, B1 and C1

At the second step, an FIR digital filter is designed by using MATLAB to allow a low frequency noise signal obtained from random source to pass through it. The magnitude response of this filter is shown in Figure 5. 


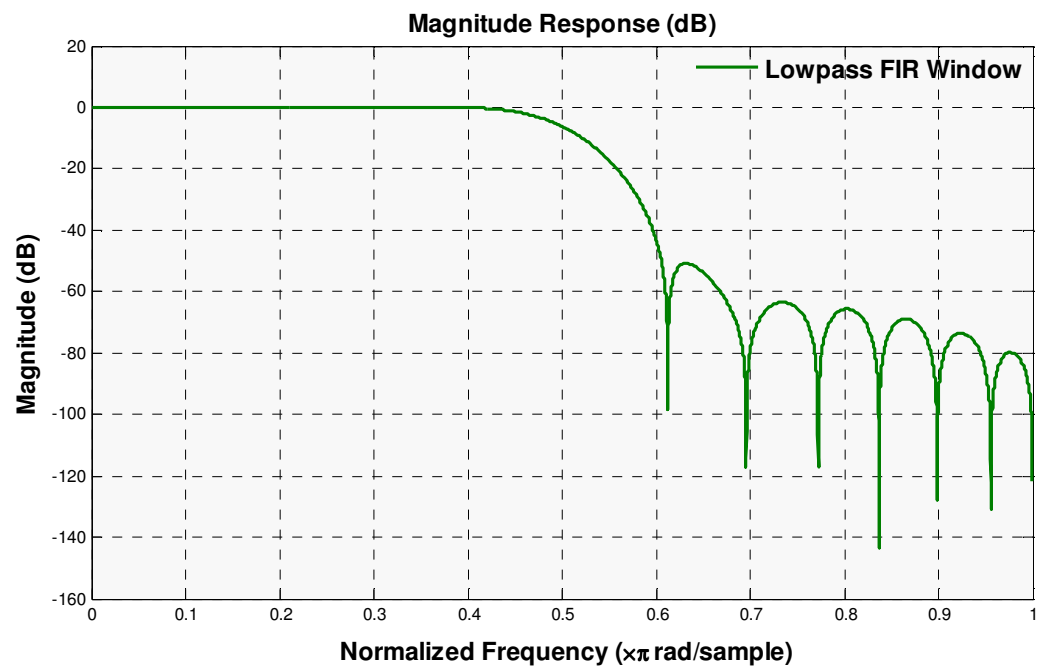

Figure 5. Magnitude response of FIR digital filter

At the third step, three FIR digital filters are designed to reconstruct the three sinusoidal signals from the composite signal. These three FIR digital filters are designed by using MATLAB. The magnitude responses of these three filters are shown in Figure 6, Figure 7 and Figure 8. Among three filter designs, the filter design1 is designed to reconstruct the sinusoidal signal A1.The filter design2 is designed to reconstruct the sinusoidal signal B2. The filter design 3 is designed to reconstruct the sinusoidal signal $\mathrm{C} 1$. All three FIR digital filters form the filter combination block. To design these three FIR digital filters, initially the filter response type is fixed (Low pass response). Then the design method is fixed (FIR window) and the window type is fixed (Hamming window). Finally the cut off frequency, the filter order and sampling frequency are fixed to get the desired magnitude response. From Figure 6, Figure 7 and Figure 8, it is seen that the attenuation at cut off frequencies is fixed at $6 \mathrm{~dB}$ (half the pass band gain) and the vertical axis represents the magnitude $(\mathrm{dB})$ and the horizontal axis represents the normalized frequency $(\times \pi$ radian/sample).

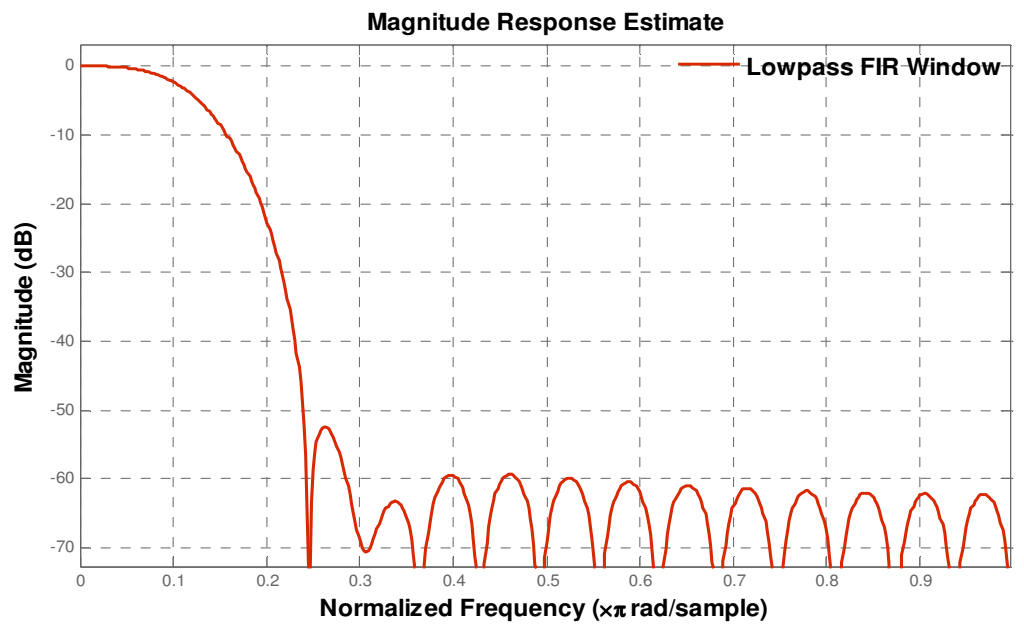

Figure 6. Magnitude response of digital filter design1 
Signal \& Image Processing : An International Journal (SIPIJ) Vol.6, No.2, April 2015

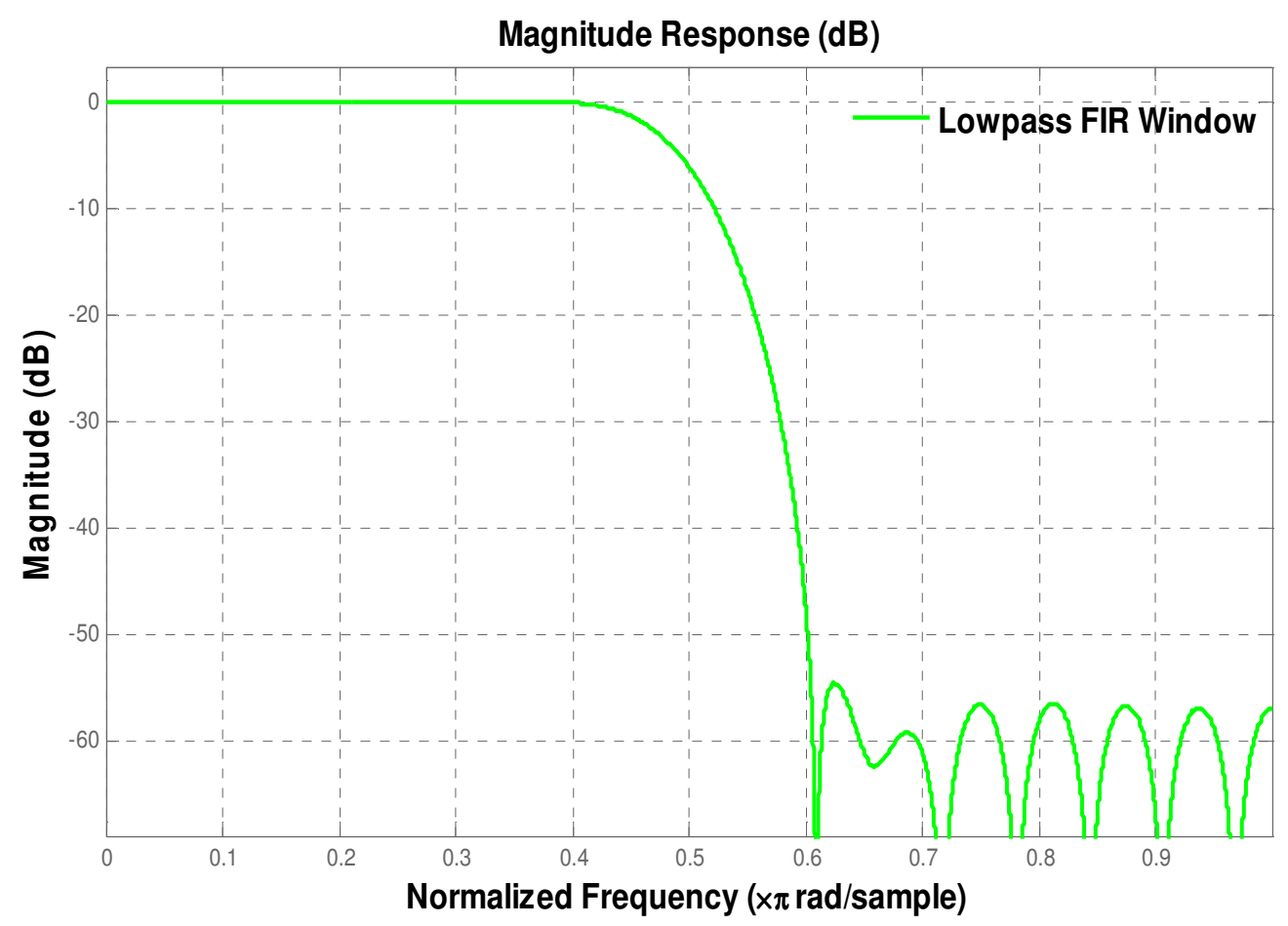

Figure 7. Magnitude response of digital filter design2

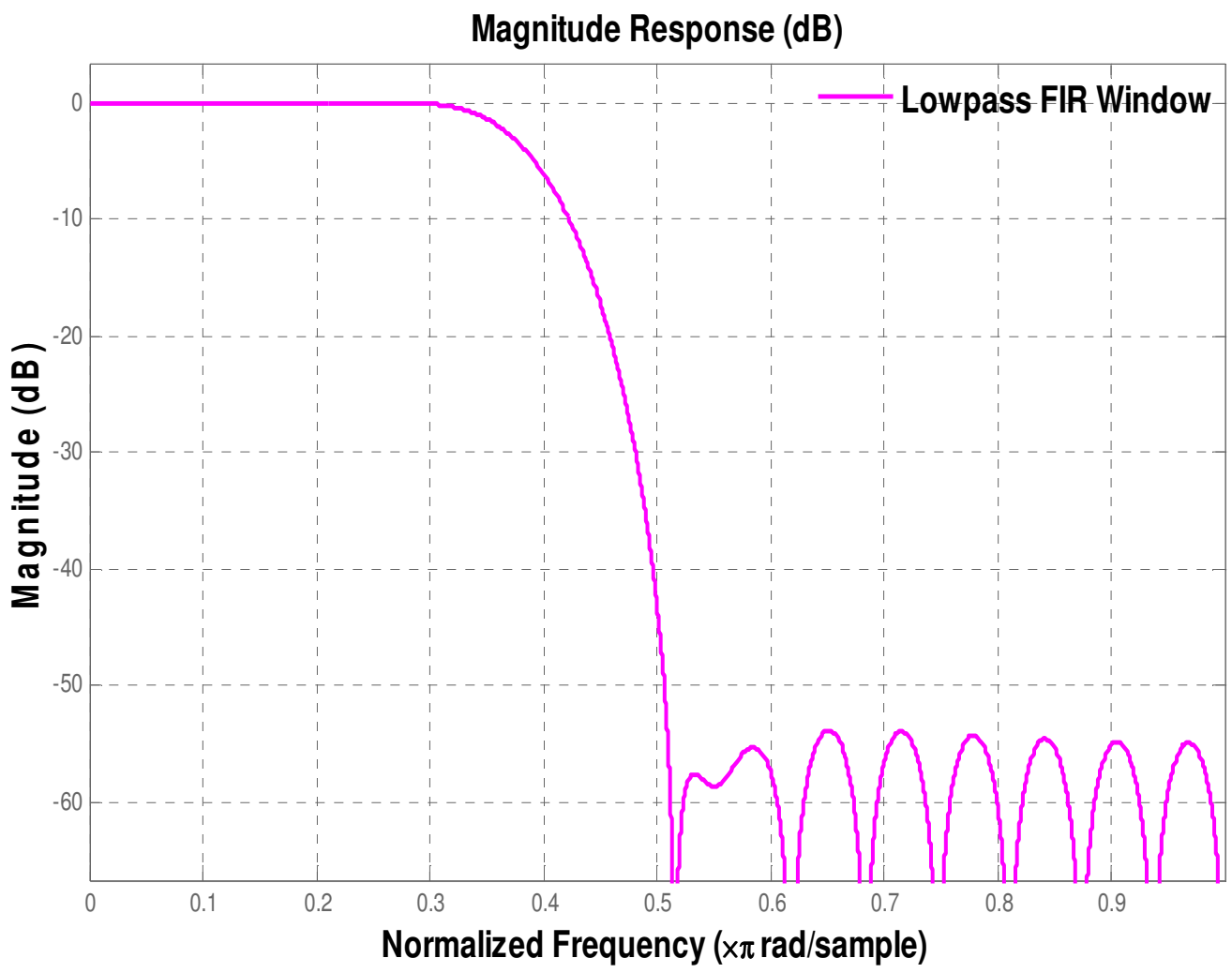

Figure 8. Magnitude response of digital filter design3 


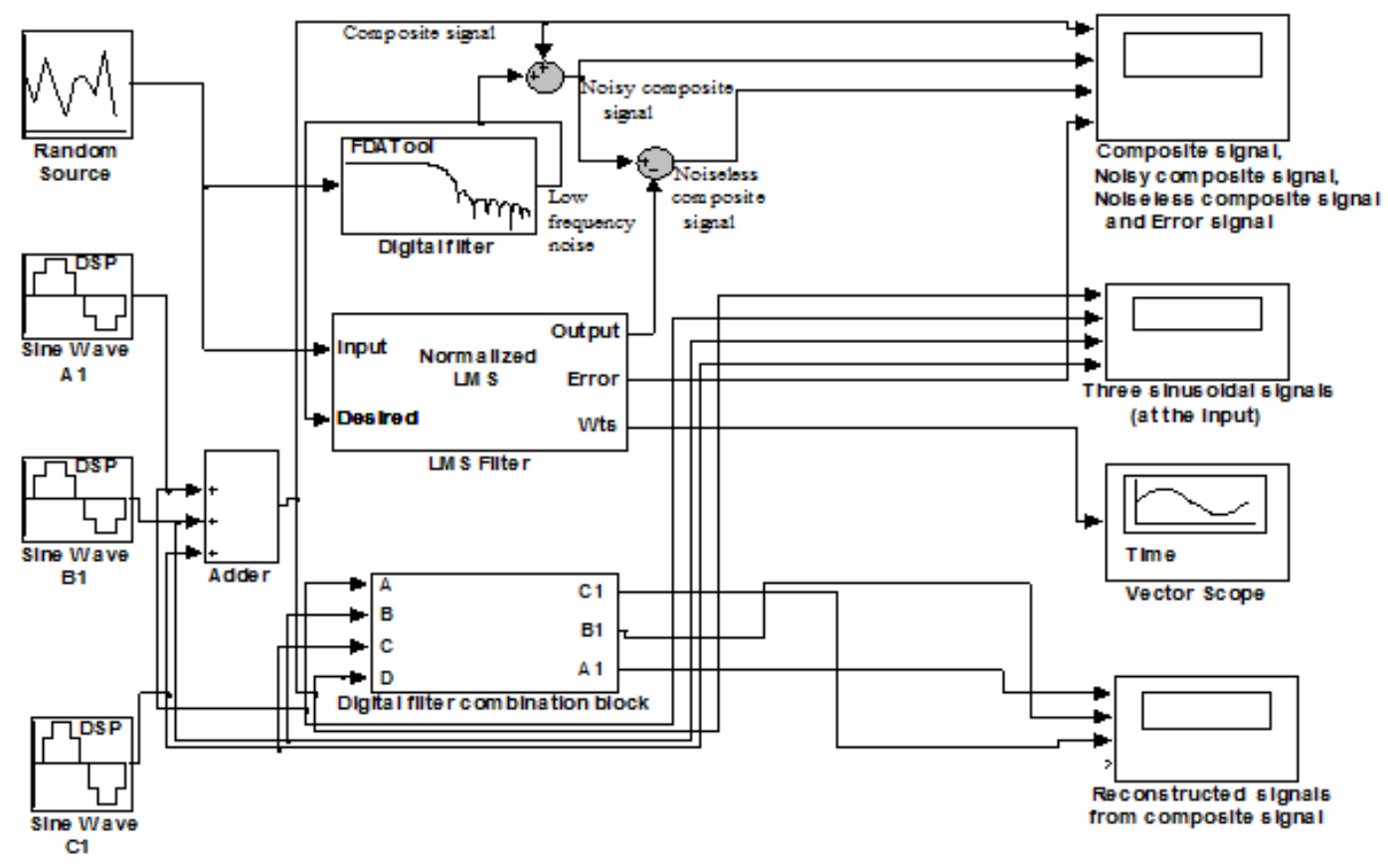

Figure 9. Simulation block diagram to reduce noise and reconstruct the input signals

\subsection{LMS FILTER}

The LMS Filter block, shown in Figure 9, can implement an adaptive FIR filter using five different algorithms. The block estimates the filter weights, or coefficients, needed to minimize the error between the output signal and the desired signal [10]. Connect the signal which I want to filter to the Input port. This input signal can be a sample-based scalar or a single-channel framebased signal. Connect the desired signal to the desired port. The desired signal must have the same data type, frame status, complexity, and dimensions as the input signal. The Output port outputs the filtered input signal, which is the estimate of the desired signal. The output of the Output port has the same frame status as the input signal. The Error port outputs the result of subtracting the output signal from the desired signal [11]. An important general form for adaptive filters is the Least Mean Square (LMS) filter. Consider the N-th order FIR filter,

$$
y_{n}=h(1) x_{n-1}+h(2) x_{n-2}+\ldots \ldots .+h(n) x_{n-\mathrm{N}}
$$

the filter error is,

$$
e(n)=x_{n}-y_{n}
$$

The LMS filter adjusts the values of the coefficients, $h$, proportional to the error, $e$

$$
h_{\mathrm{i}}(n)=h_{i-1}(n)+2 \mu x_{n-i} e_{n}
$$

where $\mu$ is a learning factor which controls how strongly the error is weighted. This equation is the consequence of the requirement to minimize mean square value of , $e$, hence the name of the filter [12]. 
Signal \& Image Processing : An International Journal (SIPIJ) Vol.6, No.2, April 2015

\subsection{LMS ALGORITHM}

The least mean squares (LMS) algorithms adjust the filter coefficients to minimize the cost function. So LMS algorithm is important because of its simplicity and ease of computation [13]. The standard LMS algorithm performs the following operations to update the coefficients of an adaptive filter:

- Calculates the output signal $y(n)$ from the adaptive filter

- Calculates the error signal $e(n)$ by using the following equation [14]: $e(n)=d(n)-y$ $(n) ; d(n)=$ desired signal

- Updates the filter coefficients by using the following equation [15]:

$$
\widehat{w}(n+1)=\widehat{w}(n)+\mu \cdot e(n) \cdot \hat{u}(n)
$$

Where $\mu$ is the step size of the adaptive filter, $\widehat{w}(n)$ is the filter coefficients vector, and $\widehat{u}(n)$ is the filter input vector. The normalized LMS (NLMS) algorithm is a modified form of the standard LMS algorithm [16]. The NLMS algorithm updates the coefficients of an adaptive filter by using the following equation:

$\widehat{w}(n+1)=\widehat{w}(n)+\mu \cdot e(n) \cdot \frac{\widehat{u}(n)}{\|\widehat{u}(n)\|^{2}}$

\subsection{FILTER DESIGN AND ANALYSIS TOOL (FDA Tool)}

The Filter Design and Analysis Tool (FDA Tool) is a powerful user interface for designing and analyzing filters swiftly. FDA Tool enables to design digital FIR or IIR filters by setting filter specifications, by importing filters from MATLAB workspace, or by adding, moving or deleting poles and zeros. FDA Tool also provides tools for analyzing filters, such as magnitude and phase response and pole-zero plots [17].

\subsection{VECTOR SCOPE}

The Vector Scope block is a comprehensive display tool similar to a digital oscilloscope. The block can display time-domain, frequency-domain, or user-defined signals. The input to the Vector Scope block can be any real-valued M-by-N matrix, column or row vector, or 1-D (Dimensional) vector, where 1-D vectors are treated as column vectors. Regardless of the input frame status, the block treats each column of an M-by-N input as an independent channel of data with M consecutive samples [18].

\subsection{OPERATION OF SIMULATION BLOCK DIAGRAM}

Consider three sinusoidal signals A1, B1 and C1 as shown in Figure6. Let the amplitudes of A1, $\mathrm{B} 1$ and $\mathrm{C} 1$ are $0.5,2$ and 5 respectively and the frequencies are $0.4 \mathrm{~Hz}, 0.25 \mathrm{~Hz}$ and $0.125 \mathrm{~Hz}$ respectively. These three sinusoidal signals are combined applying superposition principle by adder block. The output of the adder block is a composite signal. The composite signal is now applied at the input of two input sum block and the vector scope input. A random source produces a random signal (noise signal) and the random signal is applied at the input of a LMS filter. At the same time the random signal is passed through a digital filter that produces a low frequency noise signal. The low frequency noise signal is then applied at the desired input of the LMS filter and at the input of the sum block. The output of the sum block is a noisy composite signal and applied at the input of other sum block and vector scope input. The output of the LMS filter is then applied at the sum block input. The output of the sum block is the noiseless composite signal. The three sinusoidal signals are reconstructed by applying digital filter bank that is shown in Figure 10. 
Signal \& Image Processing : An International Journal (SIPIJ) Vol.6, No.2, April 2015

Finally noiseless composite signal and reconstructed signals are obtained which are shown in Figure 12 and figure 13.

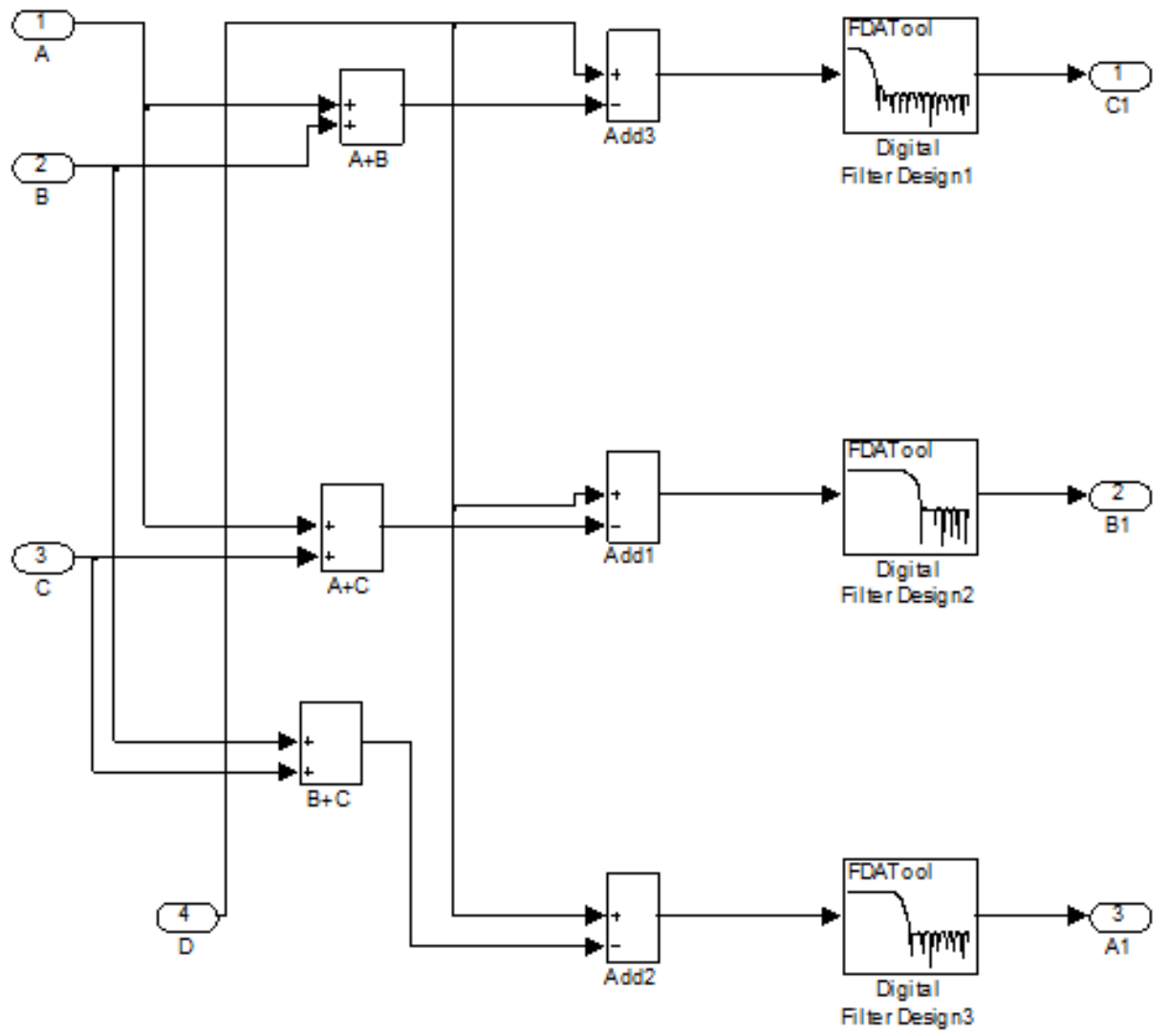

Figure 10. Filter combinations block (containing three digital FIR filters)

\section{RESULTS AND DISCUSSION}

From Figure 12, it is observed that the noiseless composite signal is perfectly obtained from noisy composite signal by using digital filter bank. So the error signal represents zero. It is also observed that the reconstructed signals are exactly same as the input sinusoidal signals (shown in figure 13). This means that the amplitudes, frequencies and shape of reconstructed signals are similar as the input sinusoidal signals. Therefore, perfect noise reduction and reconstruction are obtained. The comparison among input sinusoidal signals and reconstructed signals according to their amplitudes are shown in table 1 and table 2. From the two tables, it is observed that the amplitudes of input sinusoidal signals are exactly same as the output reconstructed signals. 
Signal \& Image Processing : An International Journal (SIPIJ) Vol.6, No.2, April 2015

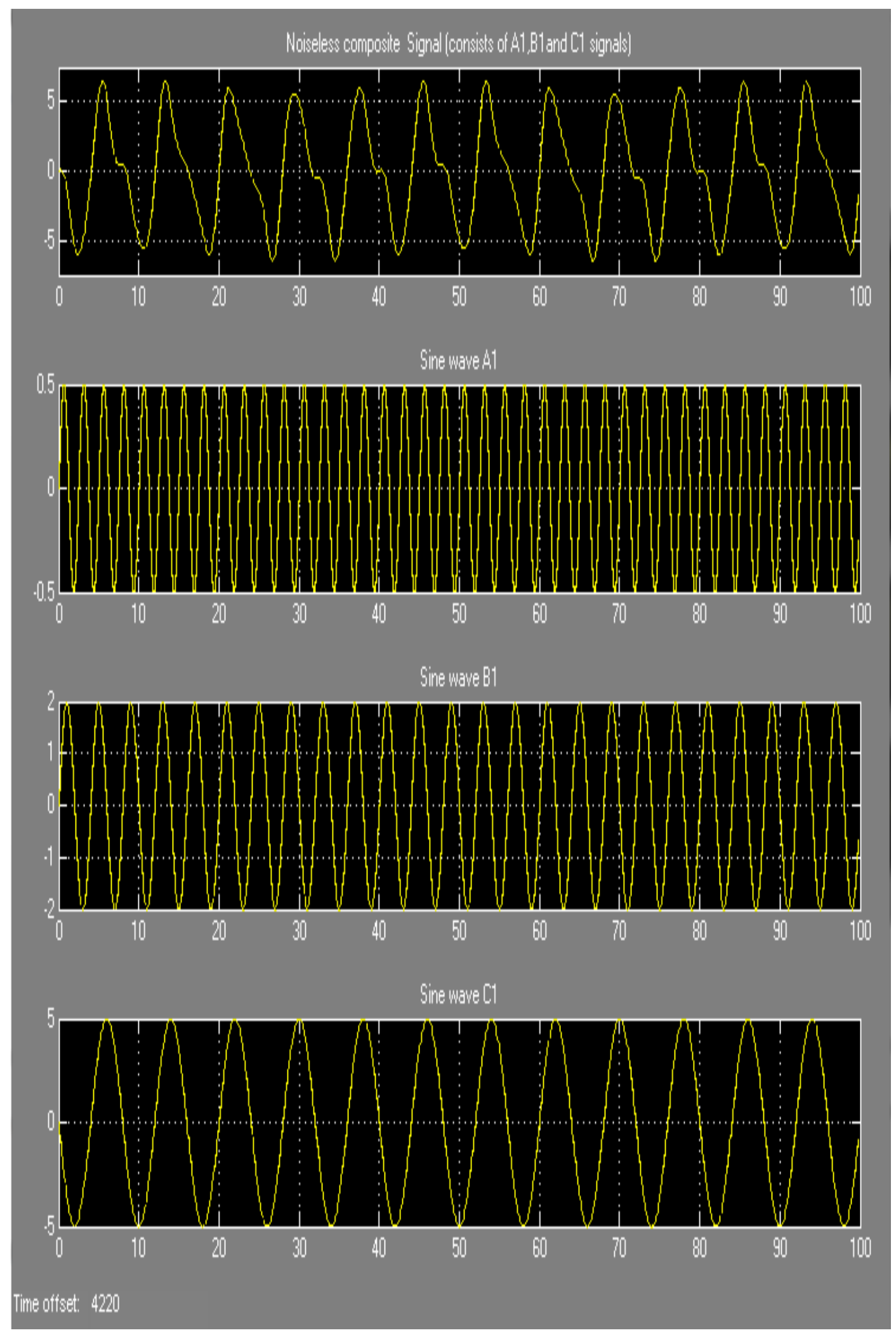

Figure 11. Composite signal (contains three input sinusoidal signals) and input sine wave A1, B1 and C1 respectively 
Signal \& Image Processing : An International Journal (SIPIJ) Vol.6, No.2, April 2015

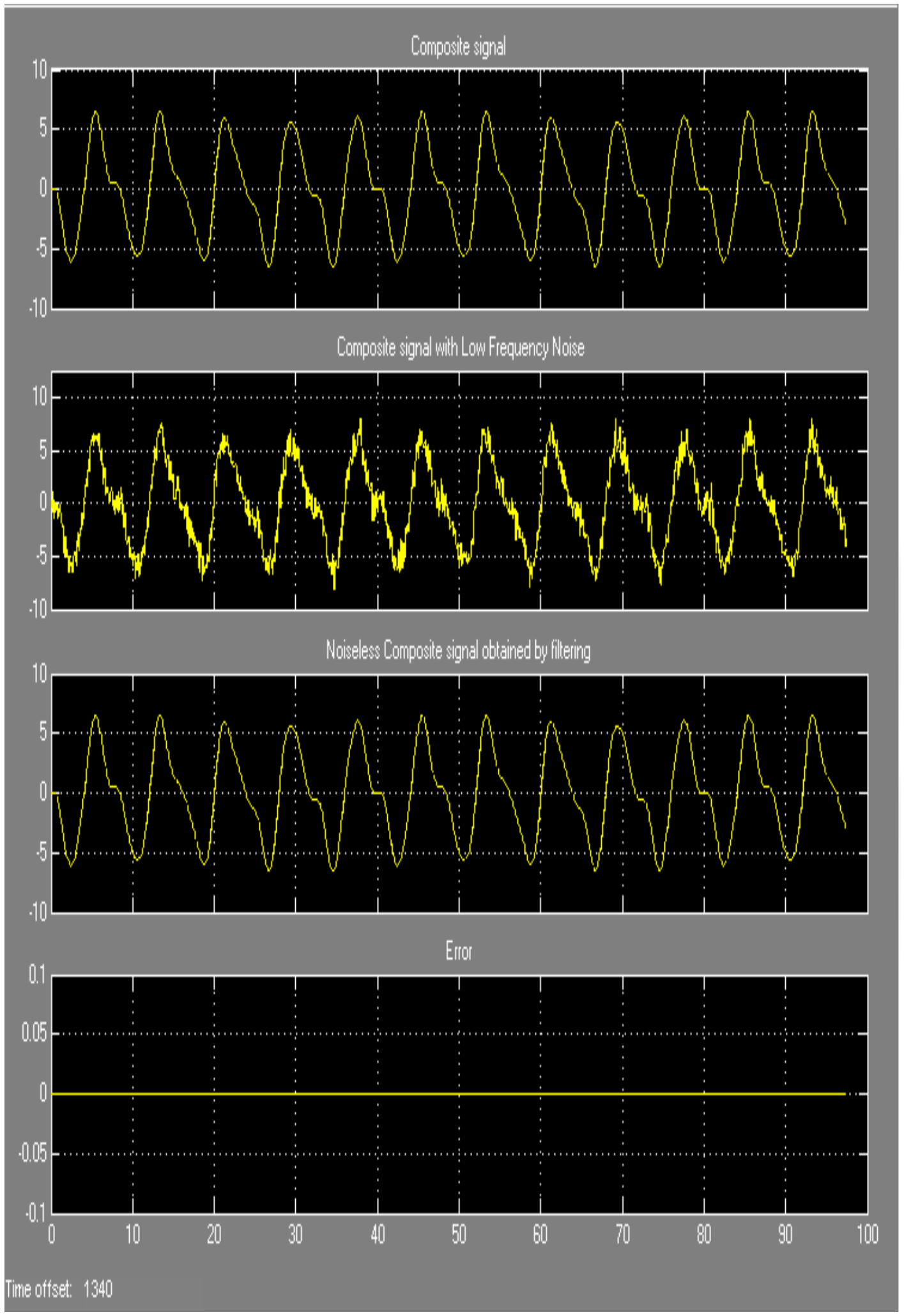

Figure 12. Composite signal, Noisy composite signal, Noiseless composite signal and Error respectively 
Signal \& Image Processing : An International Journal (SIPIJ) Vol.6, No.2, April 2015

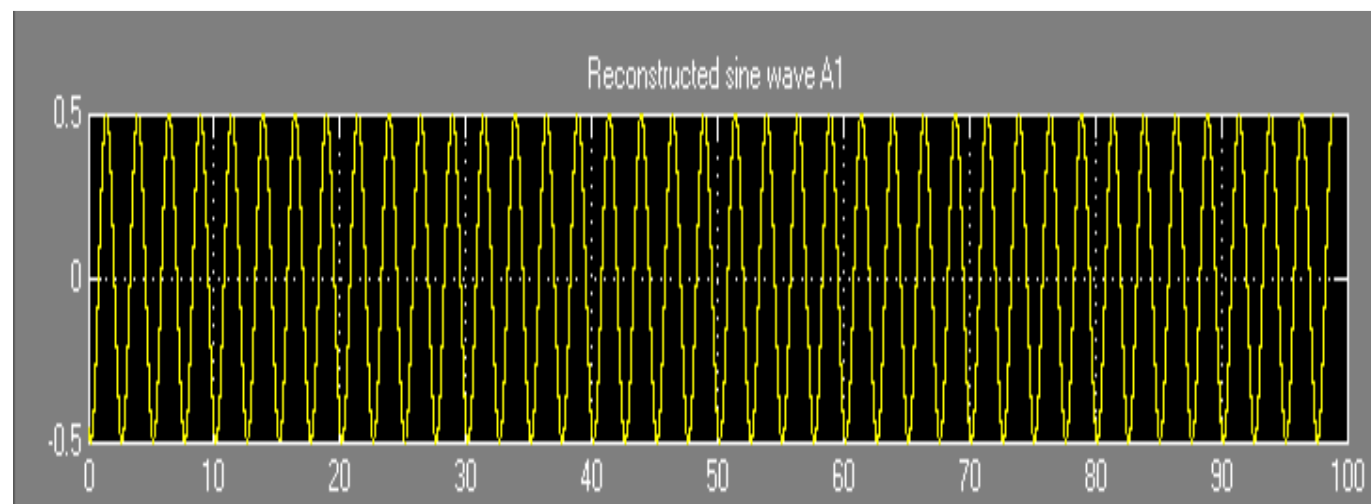

Reconstucted sine wave B1

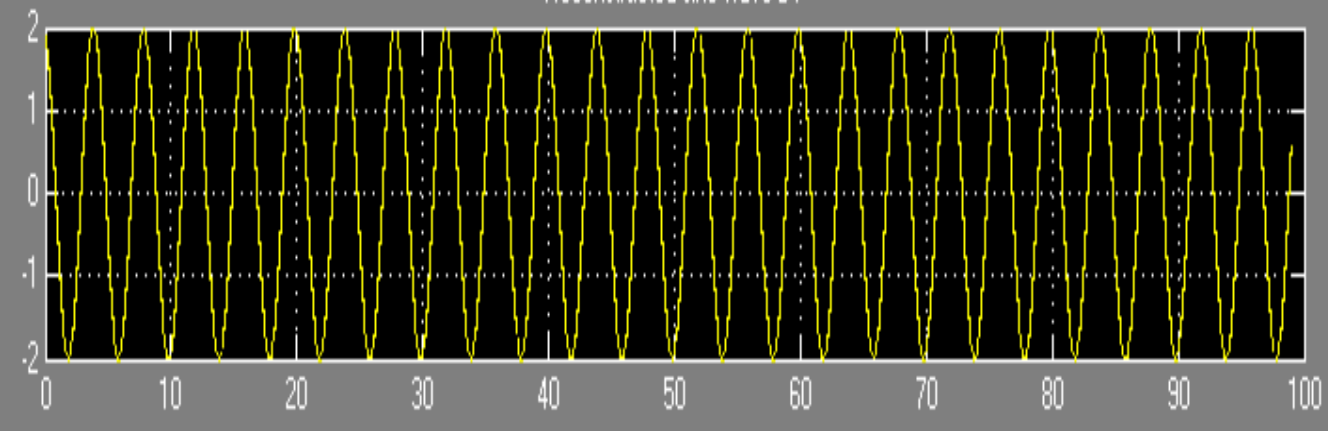

Reconstuded sine wave Cl

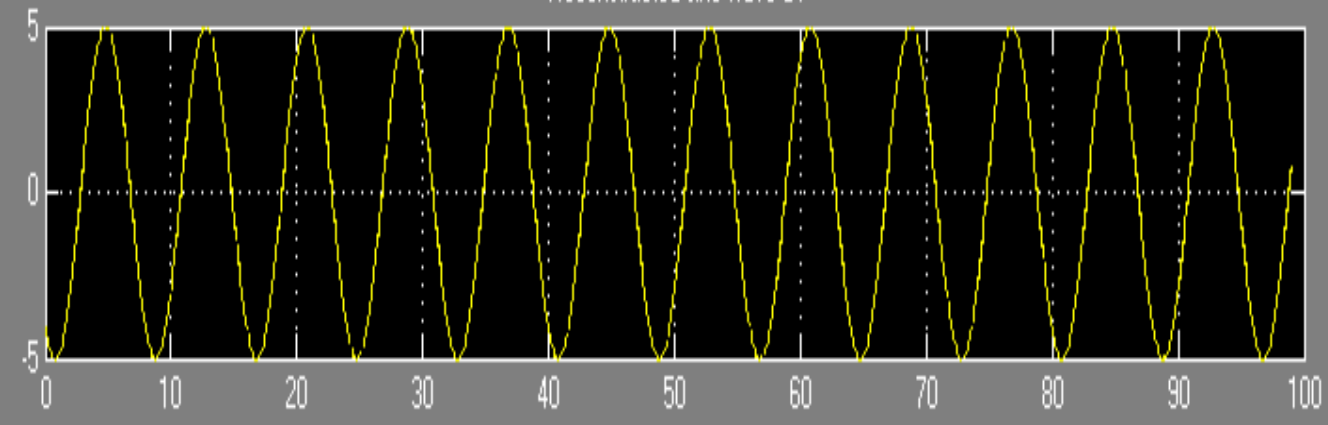

Eriol
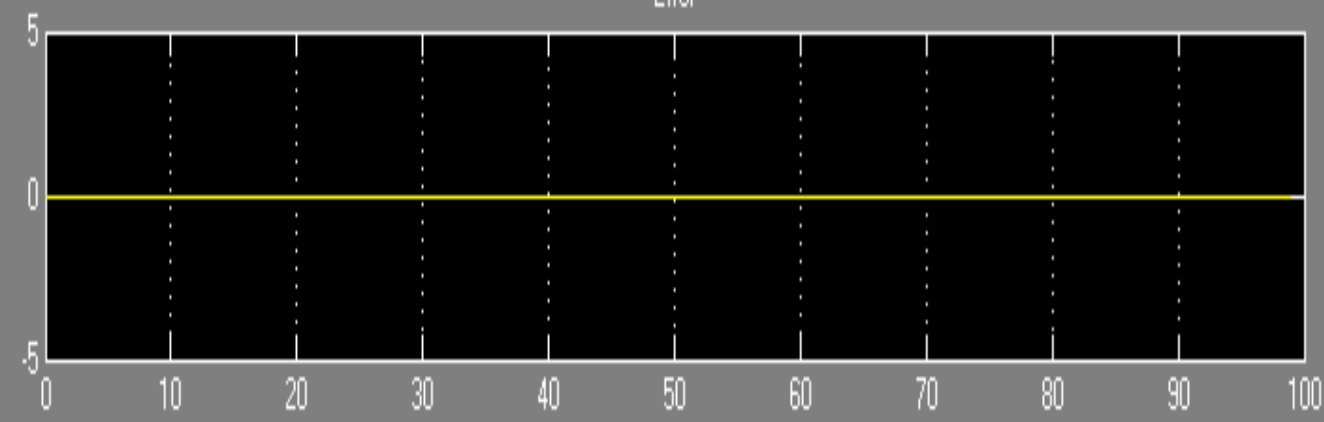

Time offset: 7750

Figure 13. Reconstructed sine wave A1, sine wave B1, sine wave C1 and zero error respectively 
Signal \& Image Processing : An International Journal (SIPIJ) Vol.6, No.2, April 2015

Table 1. Amplitudes of input sinusoidal signals

\begin{tabular}{cc}
\hline Input Sinusoidal Signals & Amplitude (volt) \\
\hline Sine Wave A1 & 0.2 \\
Sine Wave B1 & 2 \\
Sine Wave C1 & 5 \\
\hline
\end{tabular}

Table 2. Amplitudes of reconstructed signals

\begin{tabular}{rc}
\hline Reconstructed Sinusoidal Signals & Amplitude (volt) \\
\hline Reconstructed Sine Wave A1 & 0.2 \\
Reconstructed Sine Wave B1 & 2 \\
Reconstructed Sine Wave C1 & 5 \\
\hline
\end{tabular}

\section{CONCLUSiON}

Design and implementation of digital filter bank have been done in this paper to reduce noise and reconstruct the input signals. This new process represents a significant improvement over analog filters that also reduce noises. Among the different tasks of digital filter bank, only one task is shown in this paper which is noise removal. In future work, I will try to implement others work of digital filter bank and invent new work over digital filter bank.

\section{REFERENCES}

[1] A. Kumar G.K. Singh \& R. S. Anand (May 2009) "Design of Quadrature Mirror Filter Bank Using Particle Swam Optimization (PSO)", International Journal of Recent Trends in Engineering, Vol. 1, No. 3, pp. 213-214.

[2] Suverna dengar, Dr. Partha Pratim \& Bhattacharya (Jan. - March 2012) "Design and Performance Evaluation of a Quadrature Mirror Filter (QMF) Bank", International Journal of Electronics \& Communication Technology, Vol. 3, Issue 1, pp. 209-210, ISSN: 2230-7109 (online) , ISSN: 22309543 (print).

[3] Cheng, Abdulla, W.; Salcic, Z. (March 2011) "Hardware -Software Codesign of Automation Speech Recognition System for Embedded Real-Time Applications", Industrial Electronics, IEEE Transactions on, Vol. 58, No. 3, pp. 850-859, doi: 10.1109/2009.2022520.

[4]E.S Kasthuri \& A.P. James (October 2012) "Speech Filters for Speech Signal Noise Reduction", International Journal of Computer Applications (0975-8887), Vol. 55, No. 18, pp. 1-2.

[5] S. Senthurpriya \& Jagadeeswari (April 2012) "An efficient Design of Non-Uniform Filter Bank for Digital Hearing Aids”, Internaional Journal of Emerging trends in Engineering \& Development, Vol. 3, Issue 2, ISSN: 2249-6149.

[6] Alfred Mertins, "Signal Analysis: Wavelets, Filter Bank, Time Frequency, Transform and Application”, Chapter 6, pp. 144-145, copyright (C) 1999, John wiley and Sons Ltd, Print ISBN 047198626-7 Electronic ISBN 0-470-84183-4.

[7] Anamika Jain \& Aditya Goel (August 2012) "Design of M Channel Perfect Reconstruction CMFB Fiter Bank for Image Compression”, International Journal of Science \& Engineeing Research, Vol. 3, Issue 8, pp. 1-2, ISSN 2229-5518.

[8] John G. Prokis, Dimitris G. Manolakis, "In Digital Signal Processing Principles, Algorithms and Applications", Chapter 11, pp. 790-791, 4th edition.

[9] Sanjit K Mitra, "Digital Signal Processing A Coputer Based Approach", Chapter 14, pp. 806-807, 3rd edition.

[10] Asit Kumar Subudhi, Biswajit Mishra \& Mihir Narayan Mohanty (2011) "VLSI Design and Implementation for Adaptive Filter Using LMS Algorithm", International Journal of Computer \& Communication Technology ( IJCCT), Vol. 2, Issue 6, pp. 86-87.

[11] www.mathworks.com > ... > DSP Modeling > Scopes and Data Logging

[12] http://www.taygeta.com/papers/number9/node5.html 
Signal \& Image Processing : An International Journal (SIPIJ) Vol.6, No.2, April 2015

[13] Ch. Santhi Rani, Dr. P. V. Subbaiah \& Dr. K. Chennakesava Reddy (Sep.-December 2008) "LMS and RLS Algorithms for Smart Antennas in a CDMA Mobile Communication Environment", International Journal of the Computer, the Internet Management, Vol. 16, No. 3, pp. 16.

[14] A. Bhavani Sankar, D. Kumar \& K. Seethalakshmi, "Performance Study of Various Adaptive Filter Algorithms for Noise Cancellation in Respiratory Signals", Signal Processing: An International Journal ( SPIJ), Vol. 4, Issue 5, pp. 271-272.

[15] Ajjaiah H.B.M, P.V. Hunagund, Manoj Kumar Singh \& P.V. Rao. (2012) "Adaptive Variable Step Size in LMS Algorithm Using Evolutionary Programming: VSSLMSEV”, Signal Processing: An International Journal (SPIJ), Vol. 6, Issue 2, pp. 78-79.

[16] http://sine.ni.com/nips/cds/view/p/lang/en/nid/205382

[17] www.mathworks.com/products/.../examples.html?.../introfdatooldem

[18] http://www.mathworks.com/help/dsp/ref/lmsfilter.html

\section{AUTHORS}

Kawser Ahammed received B.Sc. degree in Applied Physics, Electronics \& Communication Engineering and MS degree in Applied Physics, Electronics \& Communication Engineering from University of Dhaka, Dhaka, Bangladesh, in 2011 and 2012 respectively. He has published 3 international research papers. His research interests are in the areas of Signal Processing, Image Processing and Communications. $\mathrm{He}$ is currently trying to pursue a Doctoral Degree in Electrical Engineering from USA.

Md. Ershadullah received B.Sc. degree in Applied Physics, Electronics \& Communication Engineering from University of Dhaka, Dhaka, Bangladesh, in 2011. Now he is working as a Senior System Engineer in MAKS Renewable Energy Company Limited, Bangladesh. In previous he worked as a Design \& Service Engineer in Solar Intercontinental (Solar-IC) Limited, Bangladesh.

Md. Rakebul Islam Heru received B.Sc. in Applied Physics, Electronics \& Communication Engineering from University of Dhaka, Dhaka, Bangladesh, in 2011. Now he is working as an Assistant Maintenance Engineer at IT operation \& Communication Department in Bangladesh Bank (Central Bank of Bangladesh).

Saiful Islam is a B.Sc. final year student of Electrical \& Electronic Engineering, University of Dhaka, Dhaka, Bangladesh. His research interests are in the areas of Biomedical image processing and Signal processing. At present, he is pursuing his B.Sc. final year project work in National Institute of Nuclear Medicine and Allied Sciences, BSMMU Campus, Shahbag, Dhaka, Bangladesh.
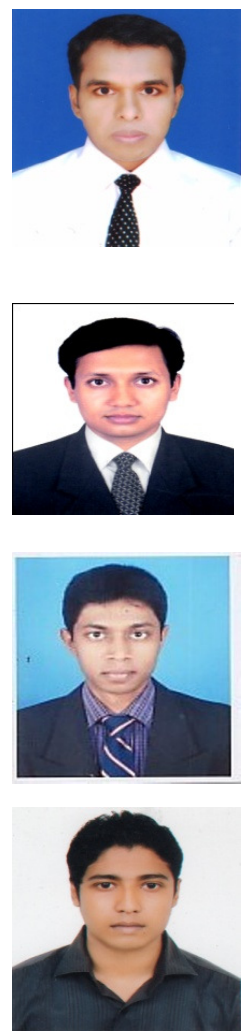